\title{
Observation of Anomalous C-O Bond Weakening on Discandium and Activation Process to CO Dissociation
}

\author{
Ling Jiang and Qiang Xu
}

\section{Supporting information}

Table 1. Comparison of Observed and Calculated IR Frequencies $\left(\mathrm{cm}^{-1}\right)$ for $\operatorname{Sc}_{2}\left[\eta^{2}\left(\mu_{2}-\mathrm{C}, \mathrm{O}\right)\right]$ and $c-\mathrm{Sc}_{2}(\mu-\mathrm{C})(\mu-\mathrm{O})$

\begin{tabular}{|c|c|c|c|c|c|c|c|c|c|}
\hline \multirow[b]{2}{*}{ Species } & \multirow[b]{2}{*}{ Mode } & \multicolumn{3}{|c|}{ Observed } & \multicolumn{4}{|c|}{ Calculated } & \multirow{2}{*}{$\begin{array}{l}\text { Scale Factor } \\
\text { (obsd/calcd) }\end{array}$} \\
\hline & & Freq $^{a}$ & ${ }^{12} \mathrm{C} /{ }^{13} \mathrm{C}$ & ${ }^{16} \mathrm{O} /{ }^{18} \mathrm{O}$ & Method & Freq $^{a}$ & ${ }^{12} \mathrm{C} /{ }^{13} \mathrm{C}$ & ${ }^{16} \mathrm{O} /{ }^{18} \mathrm{O}$ & \\
\hline \multirow[t]{2}{*}{$\mathrm{Sc}_{2}\left[\eta^{2}\left(\mu_{2}-\mathrm{C}, \mathrm{O}\right)\right]$} & $v_{\mathrm{C}-\mathrm{O}}$ & 1193.4 & 1.0237 & 1.0187 & BP86 & $1237.6(290)$ & 1.0252 & 1.0197 & 0.964 \\
\hline & & $(0.079)$ & & & BPW91 & $1240.4(298)$ & 1.0251 & 1.0198 & 0.962 \\
\hline \multirow[t]{2}{*}{$c-\mathrm{Sc}_{2}(\mu-\mathrm{C})(\mu-\mathrm{O})$} & $v_{\mathrm{Sc}-\mathrm{C}}$ & 742.6 & 1.0267 & 1.0050 & BP86 & $752.5(54)$ & 1.0260 & 1.0067 & 0.987 \\
\hline & & $(0.016)$ & & & BPW91 & $756.9(70)$ & 1.0264 & 1.0065 & 0.981 \\
\hline \multirow[t]{2}{*}{$c-\mathrm{Sc}_{2}(\mu-\mathrm{C})(\mu-\mathrm{O})$} & $v_{\mathrm{Sc}-\mathrm{O}}$ & 614.1 & 1.0020 & 1.0412 & BP86 & $601.9(115)$ & 1.0079 & 1.0365 & 1.020 \\
\hline & & $(0.028)$ & & & BPW91 & $603.3(121)$ & 1.0067 & 1.0375 & 1.018 \\
\hline
\end{tabular}

${ }^{\mathrm{a}}$ Integrated intensities for observed data and calculated intensities $(\mathrm{km} / \mathrm{mol})$ are listed in parentheses. 


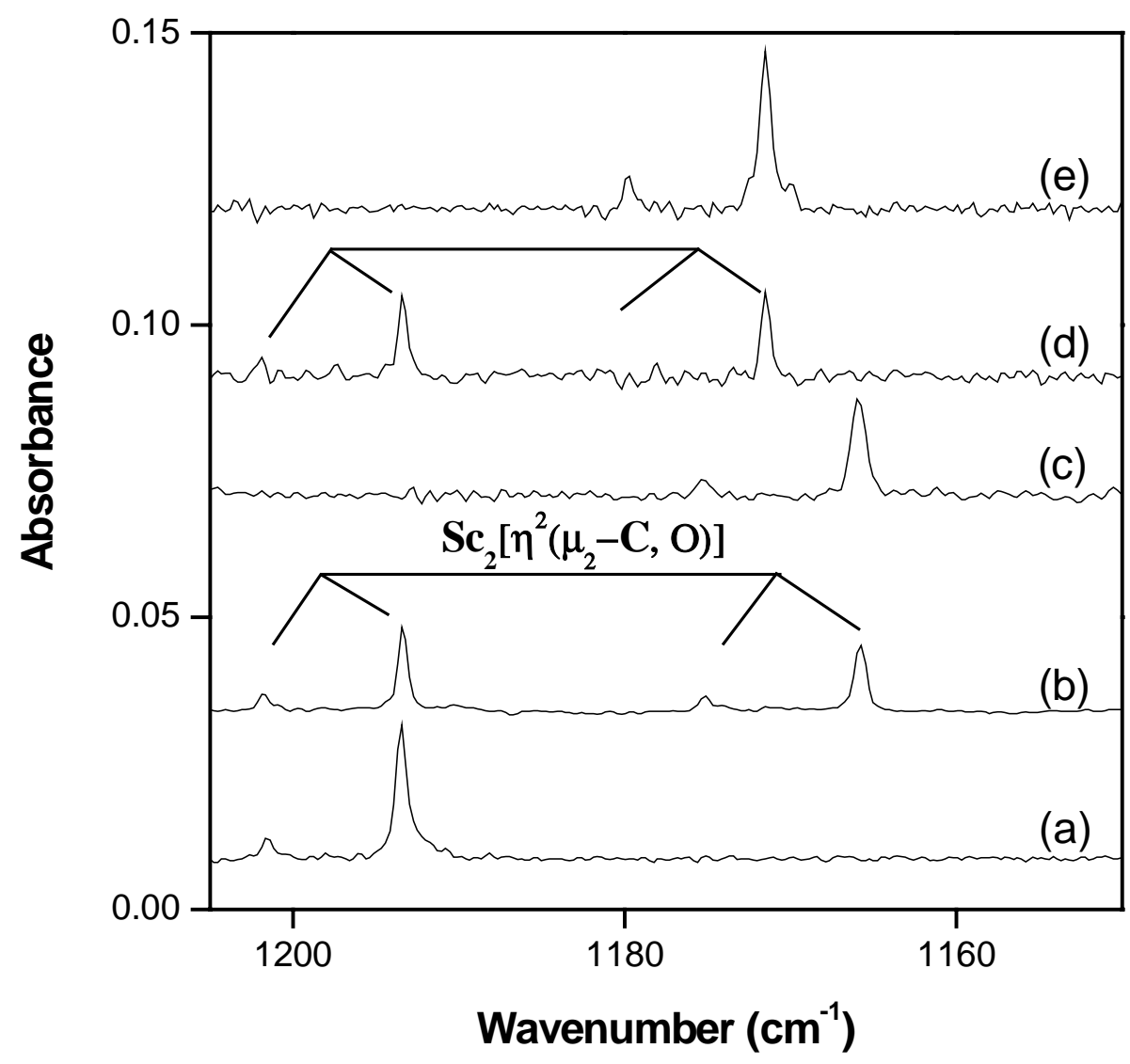

Figure 1. IR spectra in the $1210-1150 \mathrm{~cm}^{-1}$ region for laser-ablated Sc atoms co-deposited with isotopic $\mathrm{CO}$ in $\mathrm{Ar}$ for $60 \mathrm{~min}$ at $7 \mathrm{~K}$, followed by annealing to $32 \mathrm{~K}$. (a) $0.06 \%$ ${ }^{12} \mathrm{C}^{16} \mathrm{O}$, (b) $0.03 \%{ }^{12} \mathrm{C}^{16} \mathrm{O}+0.03 \%{ }^{13} \mathrm{C}^{16} \mathrm{O}$, (c) $0.06 \%{ }^{13} \mathrm{C}^{16} \mathrm{O}$, (d) $0.03 \%{ }^{12} \mathrm{C}^{16} \mathrm{O}+0.03 \%$ ${ }^{12} \mathrm{C}^{18} \mathrm{O}$, and (e) $0.06 \%{ }^{12} \mathrm{C}^{18} \mathrm{O}$. 


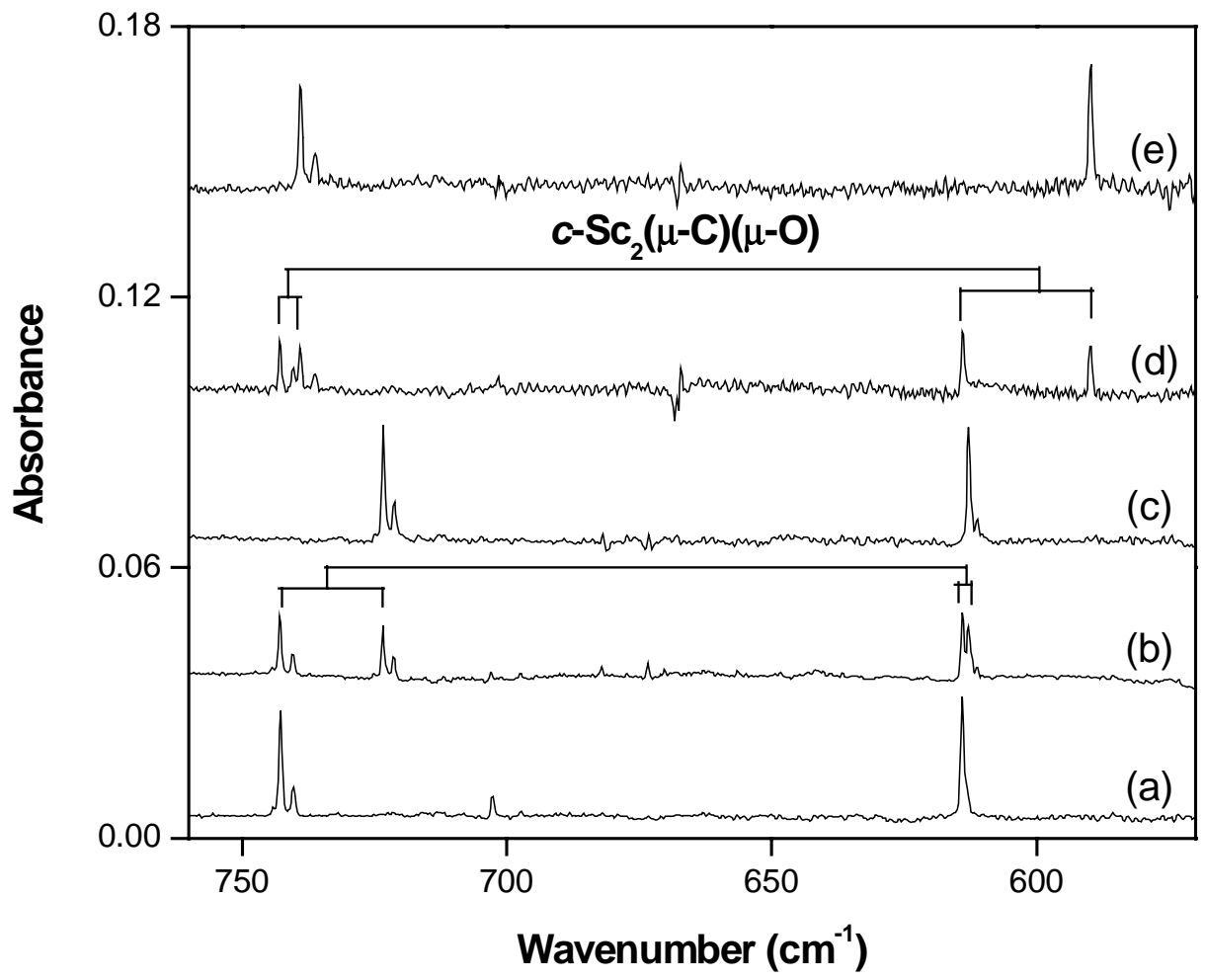

Figure 2. IR spectra in the $760-560 \mathrm{~cm}^{-1}$ region for laser-ablated Sc atoms co-deposited with isotopic $\mathrm{CO}$ in $\mathrm{Ar}$ for $60 \mathrm{~min}$ at $7 \mathrm{~K}$, followed by annealing to $32 \mathrm{~K}$ and broad-band irradiation for $20 \mathrm{~min}$. (a) $0.06 \%{ }^{12} \mathrm{C}^{16} \mathrm{O}$, (b) $0.03 \%{ }^{12} \mathrm{C}^{16} \mathrm{O}+0.03 \%{ }^{13} \mathrm{C}^{16} \mathrm{O}$, (c) $0.06 \%$ ${ }^{13} \mathrm{C}^{16} \mathrm{O}$, (d) $0.03 \%{ }^{12} \mathrm{C}^{16} \mathrm{O}+0.03 \%{ }^{12} \mathrm{C}^{18} \mathrm{O}$, and (e) $0.06 \%{ }^{12} \mathrm{C}^{18} \mathrm{O}$. 


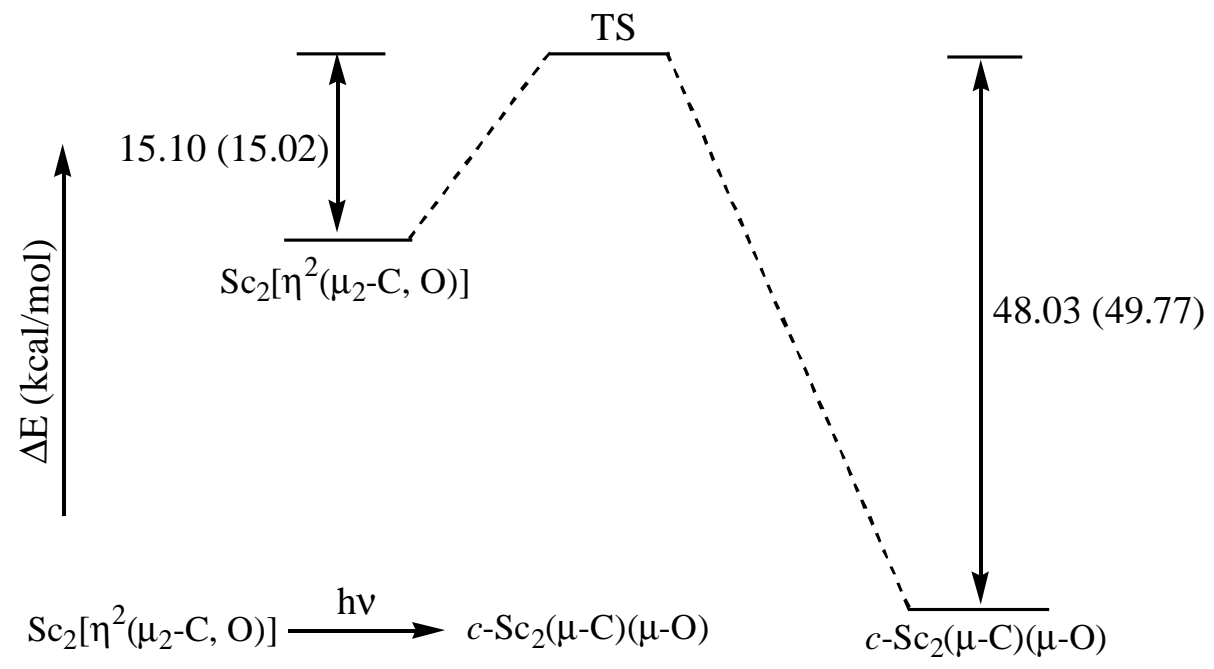

Figure 3. Potential Energy Surface (PES) for the $\mathrm{Sc}_{2}\left[\eta^{2}\left(\mu_{2}-\mathrm{C}, \mathrm{O}\right)\right] \rightarrow c-\mathrm{Sc}_{2}(\mu-\mathrm{C})(\mu-\mathrm{O})$ reaction calculated at the BP86/6-311++G(d,p) and BPW91/6-311++G(d,p) (in parentheses) levels. 